\title{
Primary Immunodeficiencies due to abnormalities of the actin cytoskeleton
}

Burns S.0. ${ }^{1,2}$, Zarafov A. ${ }^{1}$, Thrasher A. ${ }^{3,4}$.

${ }^{1}$ University College London Institute of Immunity and Transplantation, London, UK.

${ }^{2}$ Department of Immunology, Royal Free London NHS Foundation Trust, London, UK

${ }^{3}$ University College London Institute of Child Health, London, UK

${ }^{4}$ Great Ormond Street Hospital for Children NHS Foundation Trust, London, UK

Corresponding Author:

Adrian J Thrasher

Institute of Child Health

University College London

30 Guilford Street

WC1N 1EH

a.thrasher@ucl.ac.uk

Direct Telephone: +44 (0)20 79052660

PA Telephone: +44 (0)20 79052289

Fax: +44 (0)20 79052810

The work was also supported by the Higher Education Funding Council for England (SB) and The Wellcome Trust (090233/Z/09/Z, AJT) 


\section{Abstract:}

Primary Immunodeficiencies (PID) are inherited conditions where components of the immune system are missing or dysfunctional. Over 300 genes have been causally linked to monogenic forms of PID, including a number that regulate the actin cytoskeleton. The majority of cytoskeletal defects disrupt assembly and disassembly of filamentous actin in multiple immune cell lineages impacting functions such as cell migration and adhesion, pathogen uptake, intercellular communication, intracellular signalling, and cell division. In the past 24 months, new actin defects have been identified through next generation sequencing technologies. Substantial progress has also been made in understanding the pathogenic mechanisms that contribute to immunological dysfunction, and also how the cytoskeleton participates in normal physiological immune processes. This review summarises recent advances in the field, raising awareness of these conditions and our current understanding of their presentation. Description of further cases and new conditions will extend the clinical phenotype of actin relation disorders, and will promote the development of more effective and targeted therapies.

\section{Keywords:}

Actin cytoskeleton

Immune cell

Primary immunodeficiency

Autoimmunity 


\section{Introduction}

The actin cytoskeleton is required for many immune cell functions including migration, adhesion, phagocytosis, assembly of complex intercellular contacts, and cell division. More recently, a contribution of the cytoskeleton to regulation of other cell processes such as receptor signalling, calcium flux and gene expression has been appreciated, highlighting the complex multi-layered role that it can play in host defence and immune homeostasis. In the most recent international classification, 10 different primary immunodeficiencies (PID) result from defects of cytoskeletal regulation.(1) In most cases these involve actin regulators that are restricted to haematopoietic cells, which is consistent with the observation that ubiquitously expressed actin regulators are embryonically lethal in mouse models. Known cytoskeleton-associated PIDs present either as combined or severe combined immunodeficiencies (CID or SCID) or as phagocyte disorders. These monogenic conditions have taught us a great deal about the roles and regulation of actin in leukocytes. In this review, we will briefly summarise our current understanding of cytoskeletal disorders that cause PID in humans.

\section{Regulation of the actin cytoskeleton}

Dynamic rearrangement of cell shape required for motility, adhesion, particle uptake and cell division relies on rapid assembly and disassembly of filamentous actin ( F-actin, Figure 1). In steady state, F-actin polymerisation occurs spontaneously, however, the initial nucleation step, which involves the formation of $\mathrm{G}$-actin dimers and trimers, is thermodynamically unfavourable (2). For this reason, various actin nucleators exist which promote nucleation, generally in response to specific upstream signals such as integrin activation, $T$ and B-cell receptor ligation and chemokine stimulation. The three known families of actin nucleators are formins, Arp2/3 complex and WASp homology (WH2) domain-containing proteins, although the latter may only play an auxiliary role and require synergy with other factors (3). Each of the classes is differentially regulated and differs in the mechanism of action (4). Formin activity results in the generation of new unbranched filaments, which are typically associated with filopodial structures (5) while Arp2/3 complex activity results in the formation of a dense branched actin network such as that found behind the leading edge of migrating cells (6). Arp2/3 itself is a weak nucleator and requires activation by nucleation-promoting factors, such as those belonging to the Wiskott-Aldrich protein (WASp) family (7). In addition to activating the Arp2/3 complex, WASp-family members play a critical role in integrating different signals for cytoskeletal arrangement through their domain structure that enables interaction with many partners, including Rho family GTPases, phosphoinositide lipids at the plasma membrane and kinases (8). Negative regulation of F-actin assembly is equally important for normal cytoskeletal dynamics and this is achieved by several mechanisms including capping and severing of actin filaments.

\section{Combined and Severe combined immunodeficiencies}

Many haematopoietic actin regulators are expressed from early stages of cell stem differentiation and thus alterations in their function impact multiple immune lineages resulting in combined defects of humoral and cellular immunity. These are often clinically severe and most warrant correction by haematopoietic stem cell transplantation or gene therapy. The haematopoietic actin regulators described in this review are summarised in Figure 2.

\section{Wiskott Aldrich Syndrome (WAS)}

WAS is the best characterised PID resulting from defects of the actin cytoskeleton and remains the archetypical example in the field. Loss of function mutations in the WAS gene disrupt expression and 
function of the hematopoietically-restricted WAS protein (WASp) resulting in failure of Arp2/3mediated actin polymerisation. In classical WAS, where WASp expression is typically completely absent, a combined defect of innate and adaptive immunity is associated with microthrombocytopoenia, eczema, haematological malignancies and a very elevated risk of autoimmunity (9). A milder phenotype also exists (previously called X-linked thrombocytopenia), associated with preservation WASp expression, and although thrombocytopenia can be severe, immunological abnormalities are much less prominent. Interestingly, gain of function mutations result in a completely different phenotype characterised by congenital neutropenia (10).

The broad immunodeficiency seen in severe WAS is explained by widespread disruption of lymphoid and myeloid lineage immune cell functions including cell spreading, migration, phagocytosis and immune synapse assembly (reviewed in Burns and Thrasher NRI). Substantial progress has been made in understanding the role of WASp in myeloid cells and T cells for induction of T-cell responses. Recently, it was also shown that defects of dendritic cells and T-cells also result in inflammatory complications, in particular WAS-associated eczema. In an elegant study using WAS murine models, skin inflammation was demonstrated to result from a combination of failure of Langerhan cells to emigrate from the skin to lymph nodes and enhanced cross presentation of exogenous antigen resulting in ectopic activation of CD8 T-cells(11). Surprisingly, a major factor driving aberrant cross presentation is reduced acidification of phagolysosomes in WASp-deficient DC as a result of upregulated RAC2 activity in the absence of WASp. This highlights how altered function of other cytoskeletal molecules in the absence of normal WASp causes some features of the clinical disease.

A number of recent papers have highlighted the complex role of WASp in B-cells that may differ at varying stages of B-cell maturation. There is now a body of evidence demonstrating that WASpmediated actin rearrangement is necessary for $B C R$ clustering and subsequent signalling. In memory B-cells, WASp-deficiency causes impaired spreading and B-cell receptor (BCR) clustering which directly impairs BCR signalling (12). Memory B-cell activation is further disrupted by reduced transcription of the BCR co-receptor CD19, loss of CD19 recruitment of CD19 to the BCR and enhanced recruitment of the BCR negative regulators FCYRIIB and SHIP. The combined impact on memory B-cell function presumably contributes to poor antibody responses. In contrast, however, several studies have demonstrated modestly enhanced BCR responsiveness in WASp-deficient immature B-cells (13-16). It is not clear why WASp exerts different effects on BCR signalling in immature and mature B-cells but the effect in immature cells may make a significant contribution to the development of antibody mediated autoimmunity in WAS. WAS-deficient mice and humans demonstrate positive selection of self-reactive B-cells in mature naive B-cell populations, especially marginal zone B-cells (MZB). This appears to be driven by abnormally enhanced signalling through both the BCR and MyD88-dependent toll-like receptors, suggesting a role for WASp in regulation of these pathways to preserve self-tolerance. In addition to altering repertoire, excessive BCR and TLR7 signalling in WAS-deficient MZB cells promotes their egress from the MZ (15), resulting in loss of MZB cells in spleens of mice which is likely to additionally impact pathogen responses. It seems likely that altered antibody production in WAS is not only due to intrinsic B-cell defects. For example, follicular T helper cells (Tfh) play a key role in regulation B-cell antibody production in germinal centres (GC) which are poorly formed in WAS. In keeping with this observation, it was recently reported that circulating Tfh cells are reduced in patients and mice with WAS (17)as a result of reduced proliferation, increased apoptosis and altered differentiation associated with poor expression of the Tfh transcription factor BCL6.

It has been postulated that some functions of WASp are compensated for by other WASp family members, in particular the ubiquitously expressed homologue, N-WASp. Conditional knock out mice 
(in which either WASp alone or WASp and N-WASp are deleted) have revealed some important information about the interaction of WASp and N-WASp for B-cell function $(18,19)$. Additional deletion of N-WASp in WASp-deficient B-cells further impairs B-cell proliferation, GC formation and T-dependent antibody responses indicating that N-WASp partially preserves these functions in WAS. Paradoxically, however, additional deletion of N-WASp reduces IgG autoantibodies and abrogates antibody-mediated autoimmunity suggesting that unopposed activity of N-WASp in WASp-deficient B-cells is an important factor in the development of IgG antibody-mediated autoimmunity. This is relevant for patients as auto-antibody production is prevalent in both severe and mild forms of WAS (20) and N-WASp may represent a novel therapeutic target for problematic WAS-associated autoimmune disease.

\section{WASp-interacting protein (WIP)- deficiency}

Normal function of WASp depends heavily on interaction with other signalling molecules such as WIP which plays an important role in regulating WASp activation and degradation (21). Rare autosomal recessive WIP-deficiency disrupts normal WASP-WIP interactions resulting in severe reduction of cytosolic WASp(22). The cellular impact is very similar to WASp-deficiency with a more profound defect of T-cell proliferation, activation and migration compatible with additional WASpindependent functions of WIP. As very few patients with WIP-deficiency have been described, most information about the functional consequence of WIP-deficiency comes from WIP-knockout mice where, in addition to T-cell dysfunction, defects of dendritic cell podosome assembly and migration as well as B-cell migration and CD19-mediated B-cell activation have been described $(21,23,24)$.

\section{DOCK8 deficiency}

DOCK8 is a member of the DOCK family of guanine nucleotide exchange factors (GEFs) that is widely expressed and functions to regulate small G proteins including the WASp-activator CDC42. In humans DOCK8-deficiency gives rise to an autosomal recessive combined immune deficiency originally considered to be a form of Hyper-IgE syndrome $(25,26)$. Description of the largest cohort of 64 patients highlight a broad susceptibility to bacterial viral and fungal infections with variable lymphopenia and functional antibody responses (27). As DOCK8 plays a role in phosphorylation and translocation of STAT3 to the nucleus (28) it is not surprising that this condition overlaps significantly with hyper-lgE syndrome caused by autosomal dominant mutations in STAT3 where similar defects of STAT3-dependent gene expression and Th17 differentiation are evident. Despite this, DOCK8deficient patients lack a number of the typical extra haematopoietic features of STAT3 deficiency (27).

DOCK8 regulates the cytoskeleton in both myeloid and lymphoid lineages. Interestingly the impact of DOCK8 deficiency on cell migration is subtle compared WASp deficiency, mainly impacting T cells, NK-cells and dendritic cells $(29,30)$. Naive CD8 T cells are diminished and exhausted in peripheral blood of patients with DOCK8 deficiency and formation of the CD8 T cell immune synapse is impaired (31). Defects of shape and nuclear deformation lead to cell death termed cytothripsis and failure to generate long-lived skin resident memory CD8 T cells which may contribute to herpes viral skin infections (30). Similar to WASp, DOCK8 plays a role in B-cell activation particularly following TLR-9 ligation (32). T and B-cell defects are likely to be partly responsible for autoimmune manifestations as DOCK8 deficiency reduces the number and function of regulatory $T$ cells and impairs late B-cell tolerance checkpoints resulting in enrichment of autoreactive $B$ cells in mature naive B-cell compartments (33). DOCK8 has also been shown to be important for the survival and function of a number of innate immune cell subsets including classify to dendritic cells innate 
lymphoid cells, NK and NK T cells)(34-36), highlighting the breadth of the DOCK8-associated immunodeficiency.

\section{DOCK2 deficiency}

DOCK2 is a member of the DOCK-A subfamily and one of the major GEFs for Rac. Recently five unrelated patients were reported with combined immune deficiency caused by biallelic loss of function mutations in DOCK2 (37). Patients are markedly susceptible to bacterial, viral, mycobacterial and opportunistic infections including vaccine-strain viruses. T-cell lymphopenia and $T$ cell proliferative responses were universally impaired and associated with reduced Rac activation after anti-CD3 stimulation. Total $F$ actin content and chemotaxis were impaired in $T$ and $B$ cells associated with variable reduction in naive $T$ cell and $B$ cell numbers and functional antibody responses. Defects were also seen in NK cell actin polymerisation and degranulation, circulating NK T cell numbers, interferon alpha and lambda production by peripheral blood mononuclear cells and viral susceptibility of patient fibroblasts indicating a broad defect of innate immunity. DOCK2-/- mice also demonstrate defects of neutrophil chemotaxis and superoxide production (38) but these remain to be investigated in humans.

\section{Moesin (MSN) deficiency}

Recently hemizygous mutations in the moesin (MSN) gene were identified in seven boys from five different families giving rise to a novel X-linked combined immune deficiency characterised by defects of T-cell function associated with hypogammaglobulinaemia (39). MSN belongs to the ezrin, MSN, radixin (ERM) family of proteins that link actin filaments to the plasma membrane, and is important for cellular rigidity and polarisation and signalling from some surface receptors. Affected individuals suffered from recurrent bacterial infections, mainly of the respiratory tract, along with unusually severe varicella-zoster infections and molluscum contagiosum. Autoimmunity in the form of alopecia or autoantibodies leading to thrombotic thrombocytopenia purpura was seen in three patients. Curiously while MSN expression was significantly reduced in T and B cells, expression was preserved in granulocytes, monocytes and platelets. The reasons for this are not clear particularly as non-random $\mathrm{X}$ inactivation in female carriers was seen in both mononuclear and polymorphonuclear cells suggesting that the mutation impacts function in both myeloid and lymphoid lineages. The authors suggest that loss of MSN expression may increase over time or following cell activation and therefore be more prominent in long-lived lymphocytes. Impact on cellular function was demonstrated in T cells where MSN deficiency results in impaired proliferation after activation, upregulated adhesion and defective migration. Although numbers of circulating neutrophils, $\mathrm{B}$ cells and NK cells are reduced, the impact of MSN mutations on the function of these cell lineages remains to be investigated.

\section{Other PID that impact cytoskeletal function}

A number of other PID have been described arising from mutation in genes that are not directly involved in regulation of F-actin but impact specific actin-mediated responses. For example, CID resulting from loss of function mutations in MST1 (also known as STK-4) is associated with reduced adhesion and chemotaxis of lymphocytes in response to CXCL11 (40), in keeping with a known role for MST1 in mediating 'inside-out signals' from chemokines to LFA-1 integrin activation in murine lymphocytes (41). In mice, at least, this has been shown to result in hypoplastic lymphoid tissue and reduced thymic egress (41), reminiscent of Coronin-1A-deficiency (42). 
Life-threatening bacterial infections in infancy associated with neutrophilia are the hallmark of LADs which impair both leukocyte adhesion and migration. LAD1,2 and 3 have been well documented (43) and will not be discussed here. LAD IV has been proposed as a classification for Rac2deficiency,(44) a very rare autosomal recessive disorder in which loss of function Rac2 mutations cause defects of neutrophil F-actin assembly, adhesion and migration resulting in neutrophilia and devastating bacterial infection. Compared with other LAD, Rac2 has the added feature of reduced respiratory burst consistent with the role of Rac2 as a component of the NADPH oxidase complex. Even though MST1 is known to regulate LFA-1 activation and bacterial infections are a component of MST1-deficiency, no significant defects of neutrophil function have been reported in this condition and neutrophilia is not a feature (45).

\section{MKL1-deficiency}

Severe neutrophil dysfunction and recurrent serious bacterial infection caused by loss of function mutations in Megakaryoblastic leukaemia 1 (MKL1) has recently been described.(46) MKL1 is a member of the myocardin -related transcription factor (MTRF) family of proteins that regulate actin dynamics. In the inactive state they are sequestered in a cytosolic complex with $\mathrm{G}$ actin which is decoupled by Rho GTPase stimulation to release actin monomers for filament formation. MKL1 then shuttles to the nucleus where it stimulates Serum Response Facor (SRF)- mediated transcription of actin and actin cytoskeleton-related genes. MKL1 deficiency in mice is known to disrupt megakaryocyte maturation and migration resulting in thrombocytopenia $(47,48)$. The function of immune cells in MKL1-deficient mice and the functional consequences for adaptive immunity remain to be investigated.

Loss of MKL1 expression in humans significantly reduces the total levels of F-actin in both lymphoid and myeloid lineage immune cells and alters the expression of multiple cytoskeleton-related genes. This resulted in a significant reduction of both phagocytosis and migration of neutrophils in keeping with the clinical phenotype. In addition, cytoskeletal defects were observed in monocyte derived dendritic cells and fibroblasts from the patient indicating a wider role for MKL1 in immune regulation and possibly wound healing. Although only mild and intermittent thrombocytopenia was apparent in the affected patient, description of additional cases will be important to further elucidate the hematopoietic and extra haematopoietic disease phenotype.

\section{Conclusion}

Although defects of the actin cytoskeleton still account for a relatively small number of PIDs, a number of additional defects have been recently identified through wider availability of genetic sequencing. Variability in clinical phenotype can be explained by their different biological functions and patterns of gene expression, and characterisation of more patients affected by individual cytoskeletal gene mutations will provide important information for development of tailored therapies. Moreover, these new discoveries provide a unique insight into the biology of the actin cytoskeleton in human immune function and homeostasis.

Key points:

- Mutations in multiple genes encoding regulators of the actin cytoskeleton are known to cause PID

- The majority of known actin-associated PID present as combined, or severe combined, immunodeficiency or as phagocyte disorders 
- The functions of multiple immune cell lineages are impaired in actin associated-PID

- These conditions are important to inform our understanding of normal actin regulation in human immune cell lineages

- Autoimmunity is a feature of some- actin associated PID and these conditions provide insight into general mechanisms of autoimmune disease

Acknowledgements:

The work was also supported by the Higher Education Funding Council for England (SB) and The Wellcome Trust (090233/Z/09/Z, AJT) 


\section{Figure Legends}

Figure 1: F-actin assembly

Actin polymerisation is initiated when signals from cell membrane receptors and phospholipids activate Rho GTPase and WASp- family proteins. Nucleating proteins such as Arp 2/3 are recruited to initiate assembly of actin filaments from monomers; this reaction is promoted by profilin that complexes with actin monomers (G-actin) in the cytosol and converts G-actin-bound ADP to ATP. Further regulation is provided by capping proteins and severing proteins including ADF/cofilin.

Figure 2: PID-associated actin regulators

Multiple regulators of the actin cytoskeleton are implicated in primary immunodeficiency, mainly as a result of loss of function mutations in their encoding genes. The main regulators discussed in this review are shown here. DOCK proteins are activated by signals from the plasma membrane and function to convert inactive GDP-bound Rho family proteins to active GTP-bound forms. Rho-family proteins in turn activate other proteins including the actin nucleator Arp2/3 (directly and through WASp). MKL1 both regulates the pool of actin monomers available for filament assembly and gene expression of other actin-regulators. ERM and Coronin Type 1 proteins play important roles in linking assembled actin filaments to the plasma membrane for cortical rigidity and membrane protrusion. Mutations in integrins and their signalling partners MST1 and Kindlin-3 impair outside-in and insideout signalling which regulates $\mathrm{f}$-actin assembly and dynamics in response to environmental cues.

Abbreviations: ERM, ezrin radixin moesin family; Cor1, Coronin Type I family; ARP, Arp 2/3 complex; WASP, Wiskott-Aldrich Syndrome protein; WIP, WASp-interacting protein. 


\section{$\underline{\text { References }}$}

1. Picard C, Al-Herz W, Bousfiha A, Casanova JL, Chatila T, Conley ME, et al. Primary Immunodeficiency Diseases: an Update on the Classification from the International Union of Immunological Societies Expert Committee for Primary Immunodeficiency 2015. Journal of clinical immunology. 2015;35(8):696-726.

* Full classification of known PIDs in humans

2. Sept D, McCammon JA. Thermodynamics and kinetics of actin filament nucleation. Biophysical journal. 2001;81(2):667-74.

3. Dominguez R. The WH2 Domain and Actin Nucleation: Necessary but Insufficient. Trends in biochemical sciences. 2016;41(6):478-90.

4. Campellone KG, Welch MD. A nucleator arms race: cellular control of actin assembly. Nature reviews Molecular cell biology. 2010;11(4):237-51.

5. $\quad$ Faix J, Grosse R. Staying in shape with formins. Developmental cell. 2006;10(6):693-706.

6. Svitkina TM, Borisy GG. Arp2/3 complex and actin depolymerizing factor/cofilin in dendritic organization and treadmilling of actin filament array in lamellipodia. The Journal of cell biology. 1999;145(5):1009-26.

7. Rotty JD, Wu C, Bear JE. New insights into the regulation and cellular functions of the ARP2/3 complex. Nature reviews Molecular cell biology. 2013;14(1):7-12.

8. Thrasher AJ, Burns SO. WASP: a key immunological multitasker. Nature reviews Immunology. 2010;10(3):182-92.

9. Sullivan KE, Mullen CA, Blaese RM, Winkelstein JA. A multiinstitutional survey of the WiskottAldrich syndrome. The Journal of pediatrics. 1994;125(6 Pt 1):876-85.

10. Ancliff PJ, Blundell MP, Cory GO, Calle Y, Worth A, Kempski H, et al. Two novel activating mutations in the Wiskott-Aldrich syndrome protein result in congenital neutropenia. Blood. 2006;108(7):2182-9.

11. Baptista MA, Keszei M, Oliveira M, Sunahara KK, Andersson J, Dahlberg Cl, et al. Deletion of Wiskott-Aldrich syndrome protein triggers Rac2 activity and increased cross-presentation by dendritic cells. 2016;7:12175.

**Elegant study examining the pathogenesis of eczema in WAS, providing important new insights into the mechanisms of WAS-associated inflammation

12. Bai X, Zhang Y, Huang L, Wang J, Li W, Niu L, et al. The early activation of memory B cells from Wiskott-Aldrich syndrome patients is suppressed by CD19 down regulation. Blood. 2016.

13. Becker-Herman S, Meyer-Bahlburg A, Schwartz MA, Jackson SW, Hudkins KL, Liu C, et al. WASp-deficient $B$ cells play a critical, cell-intrinsic role in triggering autoimmunity. The Journal of experimental medicine. 2011;208(10):2033-42.

14. Jackson SW, Jacobs HM, Arkatkar T, Dam EM, Scharping NE, Kolhatkar NS, et al. B cell IFNgamma receptor signaling promotes autoimmune germinal centers via cell-intrinsic induction of BCL6. The Journal of experimental medicine. 2016;213(5):733-50.

15. Kolhatkar NS, Scharping NE, Sullivan JM, Jacobs HM, Schwartz MA, Khim S, et al. B-cell intrinsic TLR7 signals promote depletion of the marginal zone in a murine model of Wiskott-Aldrich syndrome. European journal of immunology. 2015;45(10):2773-9.

16. Kolhatkar NS, Brahmandam A, Thouvenel CD, Becker-Herman S, Jacobs HM, Schwartz MA, et al. Altered $B C R$ and TLR signals promote enhanced positive selection of autoreactive transitional $B$ cells in Wiskott-Aldrich syndrome. The Journal of experimental medicine. 2015;212(10):1663-77.

* Study providing new insights into WAS-associated autoimmune disease

17. Zhang X, Dai R, Li W, Zhao H, Zhang Y, Zhou L, et al. Abnormalities of follicular helper T-cell number and function in Wiskott-Aldrich syndrome. Blood. 2016;127(25):3180-91.

18. Volpi S, Santori E, Abernethy K, Mizui M, Dahlberg CI. N-WASP is required for B-cellmediated autoimmunity in Wiskott-Aldrich syndrome. 2016;127(2):216-20. 
* Study demonstrating a role for unopposed action of N-WASp in causing WAS-associated immunodeficiency that could have therapeutic importance

19. Dahlberg $\mathrm{Cl}$, Torres ML, Petersen SH, Baptista MA, Keszei M, Volpi S, et al. Deletion of WASp and N-WASp in B cells cripples the germinal center response and results in production of IgM autoantibodies. Journal of autoimmunity. 2015;62:81-92.

20. Crestani E, Volpi S, Candotti F, Giliani S, Notarangelo LD, Chu J, et al. Broad spectrum of autoantibodies in patients with Wiskott-Aldrich syndrome and X-linked thrombocytopenia. The Journal of allergy and clinical immunology. 2015;136(5):1401-4.e1-3.

21. Chou HC, Anton IM, Holt MR, Curcio C, Lanzardo S, Worth A, et al. WIP regulates the stability and localization of WASP to podosomes in migrating dendritic cells. Current biology : CB. 2006;16(23):2337-44.

22. Lanzi G, Moratto D, Vairo D, Masneri S, Delmonte O, Paganini T, et al. A novel primary human immunodeficiency due to deficiency in the WASP-interacting protein WIP. The Journal of experimental medicine. 2012;209(1):29-34.

23. Anton IM, de la Fuente MA, Sims TN, Freeman S, Ramesh N, Hartwig JH, et al. WIP deficiency reveals a differential role for WIP and the actin cytoskeleton in T and B cell activation. Immunity. 2002;16(2):193-204.

24. Keppler SJ, Gasparrini F, Burbage M, Aggarwal S, Frederico B, Geha RS, et al. Wiskott-Aldrich Syndrome Interacting Protein Deficiency Uncovers the Role of the Co-receptor CD19 as a Generic Hub for PI3 Kinase Signaling in B Cells. Immunity. 2015;43(4):660-73.

25. Engelhardt KR, McGhee S, Winkler S, Sassi A, Woellner C, Lopez-Herrera G, et al. Large deletions and point mutations involving the dedicator of cytokinesis 8 (DOCK8) in the autosomalrecessive form of hyper-IgE syndrome. The Journal of allergy and clinical immunology. 2009;124(6):1289-302.e4.

26. Zhang Q, Davis JC, Lamborn IT, Freeman AF, Jing H, Favreau AJ, et al. Combined immunodeficiency associated with DOCK8 mutations. The New England journal of medicine. 2009;361(21):2046-55.

27. Engelhardt KR, Gertz ME, Keles S, Schaffer AA, Sigmund EC, Glocker C, et al. The extended clinical phenotype of 64 patients with dedicator of cytokinesis 8 deficiency. The Journal of allergy and clinical immunology. 2015;136(2):402-12.

* Description of the largest cohort of DOCK8 patients delineating the spectrum of disease 28. Keles S, Charbonnier LM, Kabaleeswaran V, Reisli I, Genel F, Gulez N, et al. Dedicator of cytokinesis 8 regulates signal transducer and activator of transcription 3 activation and promotes TH17 cell differentiation. The Journal of allergy and clinical immunology. 2016.

29. Krishnaswamy JK, Singh A, Gowthaman U, Wu R, Gorrepati P, Sales Nascimento M, et al. Coincidental loss of DOCK8 function in NLRP10-deficient and C3H/HeJ mice results in defective dendritic cell migration. Proceedings of the National Academy of Sciences of the United States of America. 2015;112(10):3056-61.

30. Zhang Q, Dove CG, Hor JL, Murdock HM, Strauss-Albee DM, Garcia JA, et al. DOCK8 regulates lymphocyte shape integrity for skin antiviral immunity. The Journal of experimental medicine. 2014;211(13):2549-66.

* Study clarifying the role of DOCK8 in lymphocyte cytoskeletal regulation that may explain susceptibility to viral skin infections

31. Randall KL, Chan SS, Ma CS, Fung I, Mei Y, Yabas M, et al. DOCK8 deficiency impairs CD8 T cell survival and function in humans and mice. The Journal of experimental medicine. 2011;208(11):2305-20.

32. Ham H, Guerrier S, Kim J, Schoon RA, Anderson EL, Hamann MJ, et al. Dedicator of cytokinesis 8 interacts with talin and Wiskott-Aldrich syndrome protein to regulate NK cell cytotoxicity. Journal of immunology (Baltimore, Md : 1950). 2013;190(7):3661-9. 
33. Janssen E, Morbach H, Ullas S, Bannock JM, Massad C, Menard L, et al. Dedicator of cytokinesis 8-deficient patients have a breakdown in peripheral B-cell tolerance and defective regulatory T cells. The Journal of allergy and clinical immunology. 2014;134(6):1365-74.

34. Crawford G, Enders A, Gileadi U, Stankovic S, Zhang Q, Lambe T, et al. DOCK8 is critical for the survival and function of NKT cells. Blood. 2013;122(12):2052-61.

35. Singh AK, Eken A, Fry M, Bettelli E, Oukka M. DOCK8 regulates protective immunity by controlling the function and survival of RORgammat+ ILCs. Nature communications. 2014;5:4603.

36. Keles S, Jabara HH, Reisli I, McDonald DR, Barlan I, Hanna-Wakim R, et al. Plasmacytoid dendritic cell depletion in DOCK8 deficiency: rescue of severe herpetic infections with IFN-alpha $2 b$ therapy. The Journal of allergy and clinical immunology. 2014;133(6):1753-5.e3.

37. Dobbs K, Dominguez Conde C, Zhang SY, Parolini S, Audry M, Chou J, et al. Inherited DOCK2 Deficiency in Patients with Early-Onset Invasive Infections. The New England journal of medicine. 2015;372(25):2409-22.

**First description of DOCK2 deficiency in humans

38. Watanabe M, Terasawa M, Miyano K, Yanagihara T, Uruno T, Sanematsu F, et al. DOCK2 and DOCK5 act additively in neutrophils to regulate chemotaxis, superoxide production, and extracellular trap formation. Journal of immunology (Baltimore, Md : 1950). 2014;193(11):5660-7.

39. Lagresle-Peyrou C, Luce S, Ouchani F, Soheili TS, Sadek H, Chouteau M, et al. X-linked primary immunodeficiency associated with hemizygous mutations in the moesin (MSN) gene. The Journal of allergy and clinical immunology. 2016.

**First description of MSN deficiency in humans

40. Dang TS, Willet JD, Griffin HR, Morgan NV, O'Boyle G, Arkwright PD, et al. Defective Leukocyte Adhesion and Chemotaxis Contributes to Combined Immunodeficiency in Humans with Autosomal Recessive MST1 Deficiency. Journal of clinical immunology. 2016;36(2):117-22.

41. Katagiri K, Katakai T, Ebisuno Y, Ueda Y, Okada T, Kinashi T. Mst1 controls lymphocyte trafficking and interstitial motility within lymph nodes. The EMBO journal. 2009;28(9):1319-31.

42. Punwani D, Pelz B, Yu J, Arva NC, Schafernak K, Kondratowicz K, et al. Coronin-1A: immune deficiency in humans and mice. Journal of clinical immunology. 2015;35(2):100-7.

43. Hanna S, Etzioni A. Leukocyte adhesion deficiencies. Annals of the New York Academy of Sciences. 2012;1250:50-5.

44. Pai SY, Kim C, Williams DA. Rac GTPases in human diseases. Disease markers. 2010;29(34):177-87.

45. Abdollahpour H, Appaswamy G, Kotlarz D, Diestelhorst J, Beier R, Schaffer AA, et al. The phenotype of human STK4 deficiency. Blood. 2012;119(15):3450-7.

46. Record J, Malinova D, Zenner HL, Plagnol V, Nowak K, Syed F, et al. Immunodeficiency and severe susceptibility to bacterial infection associated with a loss-of-function homozygous mutation of MKL1. Blood. 2015;126(13):1527-35.

**First description of MKL-1 deficiency in humans

47. Cheng EC, Luo Q, Bruscia EM, Renda MJ, Troy JA, Massaro SA, et al. Role for MKL1 in megakaryocytic maturation. Blood. 2009;113(12):2826-34.

48. Smith EC, Thon JN, Devine MT, Lin S, Schulz VP, Guo Y, et al. MKL1 and MKL2 play redundant and crucial roles in megakaryocyte maturation and platelet formation. Blood. 2012;120(11):2317-29. 